\title{
Linking Stage-Specific Plasma Biomarkers to Gray Matter Atrophy in Parkinson Disease
}

\author{
(D)W.-C. Lin, (1DP.-L. Lee, (D) C.-H. Lu, (D) C.-P. Lin, and (D) K.-H. Chou
}

\begin{abstract}
BACKGROUND AND PURPOSE: The shortcomings of synucleinopathy-based Parkinson disease staging highlight the need for systematic clinicopathologic elucidation and biomarkers. In this study, we investigated associations of proteinopathy and inflammation markers with changes in gray matter volume that accompany Parkinson disease progression.
\end{abstract}

MATERIALS AND METHODS: We prospectively enrolled 42 patients with idiopathic Parkinson disease, subdivided into early-/latestage groups and 27 healthy controls. Parkinson disease severity and participants' functional and cognitive performance were evaluated. Peripheral plasma $\alpha$-synuclein, $\beta$-amyloid ${ }_{42}$, and tau were quantified with immunomagnetic reduction assays, and nuclear DNA by polymerase chain reaction, and regional gray matter volumes were determined by MR imaging. Statistical tests identified stage-specific biomarkers and gray matter volume patterns in the early-stage Parkinson disease, late-stage Parkinson disease, and control groups. Correlations between gray matter volume atrophy, plasma biomarkers, Parkinson disease severity, and cognitive performance were analyzed.

RESULTS: Patients with Parkinson disease had significantly elevated $\alpha$-synuclein, tau, and $\beta$-amyloid 42 levels compared with controls; nuclear DNA levels were similar in early-stage Parkinson disease and controls, but higher in late-stage Parkinson disease (all $P$ $<$.01). We identified 3 stage-specific gray matter volume atrophy patterns: 1) control $>$ early-stage Parkinson disease $=$ late-stage Parkinson disease: right midfrontal, left lingual, and fusiform gyri, left hippocampus, and cerebellum; 2) control > early-stage Parkinson disease > late-stage Parkinson disease: precentral, postcentral, parahippocampal, left superior-temporal, right temporal, right superior-frontal, and left cingulate gyri, occipital lobe, and bilateral parts of the cerebellum; 3) control = early-stage Parkinson disease $>$ late-stage Parkinson disease: left midfrontal, superior-frontal and temporal, amygdala, and posterior cingulate gyri, caudate nucleus, and putamen. We discovered stage-specific correlations among proteinopathy, inflammation makers, topographic gray matter volume patterns, and cognitive performance that accompanied Parkinson disease progression.

CONCLUSIONS: Identifying associations linking peripheral plasma biomarkers, gray matter volume, and clinical status in Parkinson disease may facilitate earlier diagnosis and improve prognostic accuracy.

ABBREVIATIONS: $\mathrm{A} \beta=\beta$-amyloid; $\mathrm{AD}=$ Alzheimer disease; $\mathrm{CASI}=$ Cognitive Abilities Screening Instrument; $\mathrm{FDR}=$ false discovery rate; GMV $=$ gray matter volume; $\mathrm{HC}=$ healthy control; $\mathrm{HY}=$ Hoehn \& Yahr; IMR = immunomagnetic reduction; MMSE = Mini-Mental State Examination; nDNA $=$ nuclear $\mathrm{DNA} ; \mathrm{PD}=$ Parkinson disease; $\mathrm{PD}_{\mathrm{E}}=$ early-stage PD; $\mathrm{PD}_{\mathrm{L}}=$ late-stage PD; $\mathrm{SE}-\mathrm{ADL}=\mathrm{Schwab}$ and England Activities of Daily Living scale; $\mathrm{T}$-tau $=$ total tau; UPDRS = Unified Parkinson's Disease Rating Scale

$\mathbf{T}$ he degree of pathology from the onset of Parkinson disease (PD) to death is conventionally graded according to Braak staging, a neuroanatomic scheme based on the presence and

Received October 11, 2020; accepted after revision March 17, 2021.

From the Departments of Diagnostic Radiology (W.-C.L.) and Neurology (C.-H.L.) Kaohsiung Chang Gung Memorial Hospital and Chang Gung University College of Medicine, Kaohsiung, Taiwan; Institute of Neuroscience (P.-L.L., C.-P.L., K.-H.C.) Department of Biomedical Imaging and Radiological Sciences (C.-P.L.), and Brain Research Center (C.-P.L., K.-H.C.), National Yang Ming Chiao Tung University, Taipei, Taiwan.

Statistical analysis was conducted by Wei-Che Lin, MD, PhD, Department of Diagnostic Radiology, Kaohsiung Chang Gung Memorial Hospital, Chang Gung University College of Medicine, 123 Ta-Pei Rd, Niao-Sung, Kaohsiung 83305, Taiwan; e-mail: u64lin@yahoo.com.tw spread of $\alpha$-synuclein deposits in the brain (Lewy pathology). ${ }^{1}$ However, the validity of this "pure" synucleinopathy-based staging approach is controversial due to inconsistencies such as

This study received funding from the Ministry of Science and Technology, (MOST 106-2314-B-182A-031-MY2 to W.-C.L.; CORPG8L0191 to W.-C.L.; and MOST 107-2221E-010-010-MY3, to K.-H.C.).

Please address correspondence to Kun-Hsien Chou, PhD, Brain Research Center National Yang Ming Chiao Tung University, 155 Linong St, Section. 2, Taipei 11221, Taiwan; e-mail: dargonchow@gmail.com

- Indicates open access to non-subscribers at www.ajnr.org

Indicates article with online supplemental data.

http://dx.doi.org/10.3174/ajnr.A7171 
discrepancies between the pathologic stage and clinical symptoms. ${ }^{2}$ Recent studies have suggested that several coexisting misfolding proteins play important roles in the pathogenesis of PD, including cortical and limbic Lewy bodies, ${ }^{3}$ neurofibrillar tangles, senile plaques, ${ }^{4}$ and microglia. ${ }^{5}$ These heterogeneous etiologic pathways present challenges to formulating a unified staging model that encapsulates the entire disease course. Therefore, the systematic evaluation of sequential pathophysiologic and clinical changes in PD and of their mutual interactions is crucial.

In addition to synucleinopathy, it has been reported that cytotoxic intermediates of $\beta$-amyloid (A $\beta$ ) fibril formation and tau protein aggregates lead to neuronal disruption and death, while also causing symptoms commonly observed in Alzheimer disease (AD). ${ }^{6}$ Postmortem studies have confirmed the coexistence of $\mathrm{AD}$-related misfolding proteins from early-to-late stage $\mathrm{PD}$, indicating that rapid cognitive decline predicts poor prognosis in early PD. ${ }^{7,8}$ Furthermore, studies of CSF and serum biomarkers that indicate both Lewy body and $\mathrm{AD}$ pathology have garnered considerable interest. ${ }^{9-11}$ Although CSF $\mathrm{A} \beta$ and tau levels in patients with $\mathrm{PD}$ have been reported to independently predict cognitive decline, ${ }^{12}$ data are inconclusive. ${ }^{13}$ The results of studies of the total $\alpha$-synuclein level in CSF and peripheral plasma are also controversial. ${ }^{10,11}$ Explanations for such uncertainty may include, but are not limited to, methodologic variables and heterogeneous disease stages across participants in different studies. Moreover, levels of $\alpha$-synuclein are exceptionally low in plasma compared with CSF, thereby limiting the capability of enzymelinked immunosorbent assays to accurately detect plasma $\alpha$-synuclein. ${ }^{14}$ More recently, immunomagnetic reduction (IMR), which has higher sensitivity than the conventional enzyme-linked immunosorbent assay, ${ }^{11}$ has been applied to assay $\mathrm{A} \beta \mathrm{s}$, tau, and $\alpha$-synuclein in human plasma ${ }^{11,15,16}$ and to confirm PD and AD diagnoses. ${ }^{11,17,18}$ These results suggest that IMR may help to clarify the relationship between different misfolding proteins and the progression of PD.

Apart from proteinopathy, inflammation in the CNS and peripheral blood could also contribute to neurodegeneration in PD. ${ }^{19}$ Cross-talk between the brain and peripheral inflammatory markers has been implicated in numerous psychologic, behavioral, and physiologic processes. ${ }^{20}$ However, research into the association between systemic inflammation and disease progression and their interactions with proteinopathy contributing to regional gray matter volume (GMV) changes is limited and warrants further investigation.

In the present study, we systematically investigated the complex associations among multiple plasma proteinopathies, $\mathrm{A} \beta_{42}$, total tau (T-tau), and $\alpha$-synuclein; nuclear DNA (nDNA), as a marker of inflammation; and regional GMV changes during PD progression. We aimed to identify stage-specific patterns of plasma biomarkers and regional GMVs and stage-specific associations linking GMV patterns, biomarker levels, and clinical status.

\section{MATERIALS AND METHODS \\ Participants}

From August 1, 2017, to July 31, 2018, we prospectively enrolled 42 patients with a definitive diagnosis of idiopathic PD (Online Supplemental Data). ${ }^{21}$ Disease severity and functional status were evaluated in the "Off" state using the Unified Parkinson's Disease Rating Scale (UPDRS), ${ }^{22}$ the modified Hoehn \& Yahr staging scale (HY stage), ${ }^{23}$ and the Schwab and England Activities of Daily Living scale $(\mathrm{ADL})^{24}$ The Mini-Mental State Examination $(\mathrm{MMSE})^{25}$ and Cognitive Abilities Screening Instrument $(\mathrm{CASI})^{26}$ were used to assess general cognitive function. In addition, 27 sex- and age-matched healthy controls (HCs) without a medical history of neurologic or psychiatric disorders, alcohol/substance abuse, or head injury with similar education levels were enrolled.

The hospital Ethics Committee on Human Research approved the study protocol (No. 201601519B0) by Chang Gung Medical Foundation Institutional Review Board. All participants or their legally designated proxies provided written informed consent. To investigate the effects of disease stage on plasma biomarker levels and topographic GMV patterns, we further subdivided patients into 2 groups: early-stage $\mathrm{PD}\left(\mathrm{PD}_{\mathrm{E}}, \mathrm{HY}\right.$ stage $\left.\leq 2\right)$ or late-stage $\mathrm{PD}\left(\mathrm{PD}_{\mathrm{L}}, \mathrm{HY}\right.$ stage $\left.>2\right)$.

\section{Blood Sampling and Plasma Biomarker Assays}

Plasma A $\beta_{42}$, T-tau, $\alpha$-Synuclein, and nDNA. At the time of diagnosis of idiopathic $\mathrm{PD}$, potential participants were informed of the study, and those who consented were asked to provide a $10-\mathrm{mL}$ venous blood sample. The levels of multiple proteinopathies and nDNA were quantified using IMR and real-time polymerase chain reaction techniques, respectively. The processing procedures of plasma biomarker assays have been described in our previous studies $^{11,18,27-29}$ and documented in the Online Supplemental Data.

\section{Structural MR Image Acquisition and Analyses}

All participants underwent MR imaging within 1 week of peripheral plasma evaluation using a single 3T Signa (GE Healthcare) whole-body MR imaging system with an 8-channel phased array head coil. Whole-brain T1-weighted images were acquired using an axial 3D inversion recovery-prepared fast-spoiled gradient recalled-echo pulse sequence $(\mathrm{TR} / \mathrm{TE} / \mathrm{TI}=9.5 / 3.9 / 450 \mathrm{~ms}$; flip angle $=15^{\circ}$; number of excitations $=1 ; \mathrm{FOV}=24 \mathrm{~cm}$; matrix size $=512 \times 512$; voxel size $=0.47 \times 0.47 \times 1.3 \mathrm{~mm}^{3} ; 110$ contiguous slices without interslice gap and interpolation). To calculate individual regional GMVs, we first preprocessed MR images using the voxel-based morphometry approach and then calculated them with the composite anatomic atlas. The detailed image-preprocessing pipeline is documented in the Online Supplemental Data. Finally, the regional residualized GMVs, which regressed out the nuisance effects of age, sex, and total intracranial volume, were obtained.

\section{Statistics}

All statistical analyses were conducted using SPSS, Version 22.0 (IBM) and Matlab, Version R2010a (MathWorks). Figure 1 shows the analytic workflow, while details of the corresponding image and statistical analyses are as follows: 1) The ANOVA test, $\chi^{2}$ test, and ANCOVA were used to compare demographic variables and clinical evaluations between the $\mathrm{HCs}$ and the $\mathrm{PD}_{\mathrm{E}} / \mathrm{PD}_{\mathrm{L}}$ groups. Statistical significance was defined as a 2 -tailed $P$ value $<$ .05. All the continuous and categoric variables are presented as means and numbers, respectively. 2) For peripheral plasma biomarkers, we used the general linear model to adjust for the 


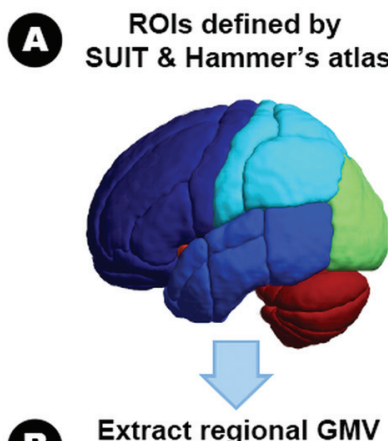

B

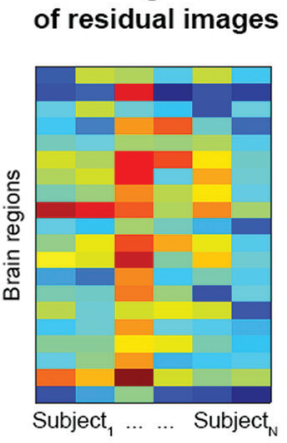

c

Pattern identification

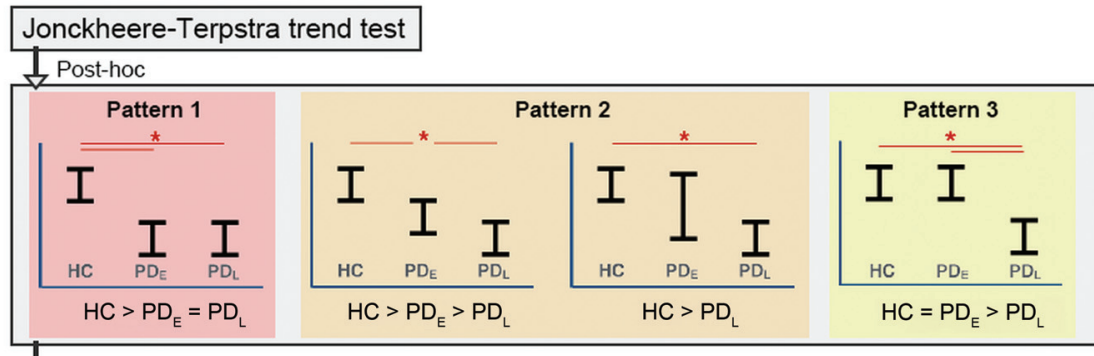

$\frac{1}{1}$

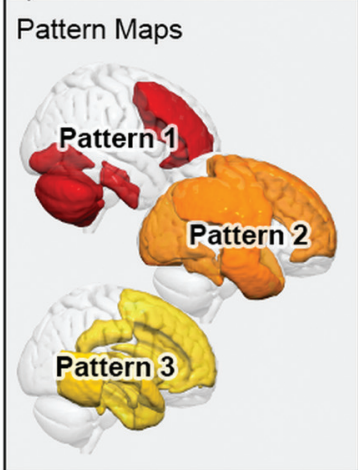

D Extract individual pattern scores by
principle component analysis
음

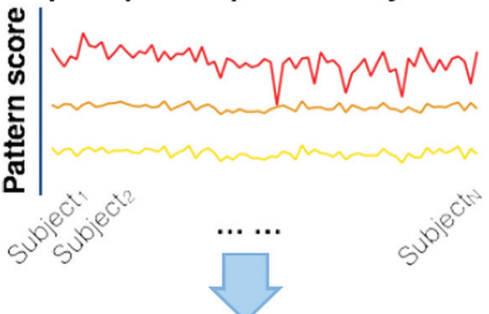

E Association with protein measurements and disease severity

FIG 1. Analytic workflow. A, Whole-brain composite atlas generated from the SUIT and Hammer's Maximum Probability Atlas. $B$, Regional gray matter volume extracted with the composite atlas. $C$, The Jonckheere-Terpstra test and pair-wise Mann-Whitney $U$ test are used to identify stage-specific patterns. $D$, Principal component analysis is used to extract weighting scores of the stage-specific GMV pattern. $E$, Spearman rank-order correlation tests are used to examine the potential associations among weighting scores of the stage-specific GMV pattern, peripheral plasma biomarkers, general cognitive performance, and disease severity. SUIT indicates Spatially Unbiased Infratentorial Template.

confounding effects of age and sex. The resulting residual value of each peripheral plasma biomarker was used for further analysis. We hypothesized that changes in peripheral plasma biomarkers and regional GMVs are associated with disease severity that would be most pronounced in patients with $\mathrm{PD}_{\mathrm{L}}$ and intermediate in those with $\mathrm{PD}_{\mathrm{E}}$ compared with $\mathrm{HCs}$. We, thus, used the nonparametric Jonckheere-Terpstra test to determine the ordered differences among the 3 study groups. The significance threshold for the nonparametric Jonckheere-Terpstra test was set at the false discovery rate (FDR)-corrected $P$ value $<.05 .{ }^{30} 3$ ) Having established possible ordered differences in peripheral plasma biomarkers and regional GMVs, we then used pair-wise Mann-Whitney $U$ tests to subdivide changing patterns of plasma biomarkers and regional GMVs into 3 categories each: plasma biomarkers, pattern 1 (control $<\mathrm{PD}_{\mathrm{E}}=\mathrm{PD}_{\mathrm{L}}$ ); pattern 2 (control $<\mathrm{PD}_{\mathrm{E}}<\mathrm{PD}_{\mathrm{L}}$ and control $<\mathrm{PD}_{\mathrm{L}}$ ); pattern 3 (control = $\mathrm{PD}_{\mathrm{E}}<\mathrm{PD}_{\mathrm{L}}$ ); and $\mathrm{GMV}$, pattern 1 (control $>\mathrm{PD}_{\mathrm{E}}=\mathrm{PD}_{\mathrm{L}}$ ); pattern 2 (control $>\mathrm{PD}_{\mathrm{E}}>\mathrm{PD}_{\mathrm{L}}$ and control $>\mathrm{PD}_{\mathrm{L}}$ ); pattern 3 (control $=\mathrm{PD}_{\mathrm{E}}>\mathrm{PD}_{\mathrm{L}}$ ). This analysis revealed a high degree of collinearity between the GMV of different anatomic regions within the same stage-specific pattern; therefore, we used principal component analysis to identify the component accounting for the largest variances of GMVs in each of the 3 stage-specific patterns and calculated its weighting score for each participant. 4) Finally, we used nonparametric Spearman rank-order correlation tests to investigate associations among weighting scores of stage-specific GMV changes, peripheral plasma biomarkers, general cognitive ability, and disease severity. ${ }^{31}$ The significance threshold for the nonparametric Spearman rank-order correlation tests was set at the FDR-corrected $P$ value $<.05$.

\section{RESULTS}

\section{Demographic and Clinical Characteristics}

The study cohorts included 42 patients with $\mathrm{PD}\left(26 \mathrm{PD}_{\mathrm{E}}\right.$ and 16 $\mathrm{PD}_{\mathrm{L}}$ ) and $27 \mathrm{HCs}$. The demographic and clinical data for each group are shown in the Online Supplemental Data. Each group had a similar average age and male/female ratio. The HCs had significantly better MMSE and CASI than patients with $\mathrm{PD}_{\mathrm{E}}$ and $\mathrm{PD}_{\mathrm{L}}$ (mean [SD], MMSE: 28.0 [1.5] versus 25.3 [3.7] versus 23.6 [5.1], respectively, $P<.001$; CASI: 92.1 [3.9] versus 81.6 [14.3] versus 78.7 [14.2], $P<.001$ ). The patients with $\mathrm{PD}$ had lower total GMV and higher CSF volume compared with HCs while the patients with $\mathrm{PD}_{\mathrm{L}}$ had lower total GMV compared with the those with $\mathrm{PD}_{\mathrm{E}}$.

\section{Stage-Specific Plasma Biomarker Patterns}

The Jonckheere-Terpstra test results retained all plasma biomarkers for post hoc tests that confirmed significantly higher $\alpha$-synuclein, T-tau, and $\mathrm{A} \beta_{42}$ levels in patients with PD compared with the HCs (Fig $2 A-C$, Online Supplemental Data), but no difference between $\mathrm{PD}_{\mathrm{E}}$ and $\mathrm{PD}_{\mathrm{L}}$, corresponding to pattern 1 (Control $<\mathrm{PD}_{\mathrm{E}}=\mathrm{PD}_{\mathrm{L}}$ ). In contrast, plasma nDNA levels were significantly higher in $\mathrm{PD}_{\mathrm{L}}$ than in $\mathrm{PD}_{\mathrm{E}}$ or controls, but similar between controls and $\mathrm{PD}_{\mathrm{E}}$, corresponding to pattern 3 (control = $\mathrm{PD}_{\mathrm{E}}<\mathrm{PD}_{\mathrm{L}}$ ) (Fig 2D, Online Supplemental Data). 
Pattern 1

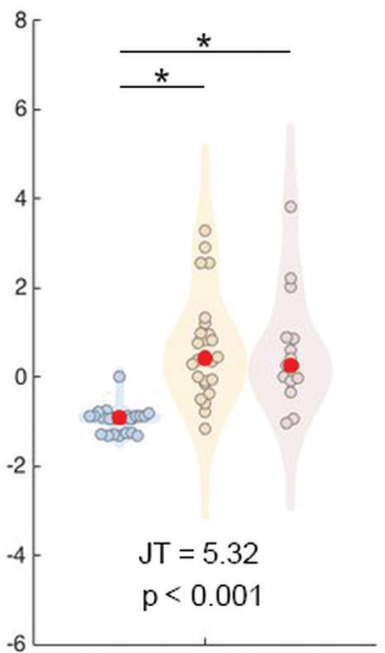

A a-synuclein
Pattern 1

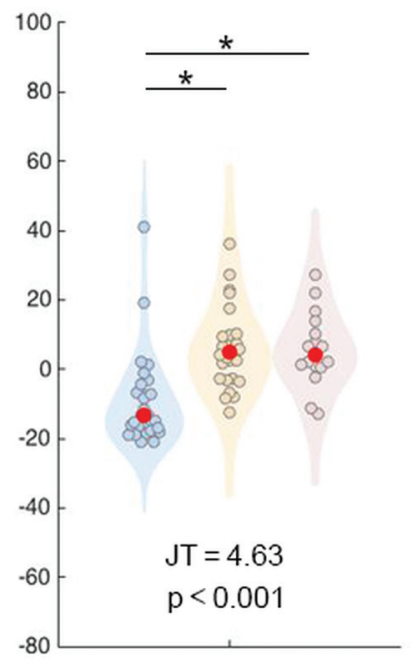

B
Pattern 1

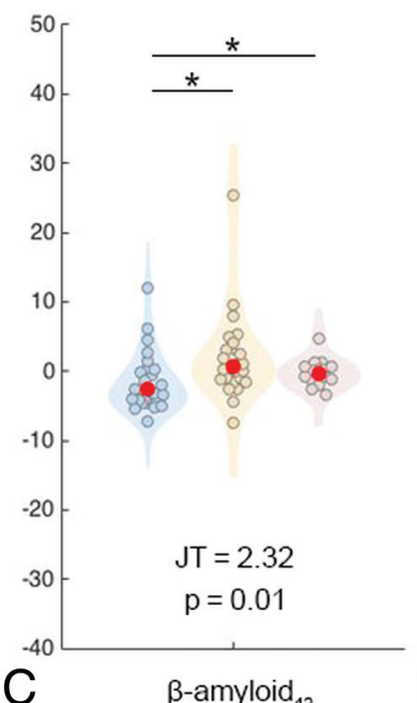

$\beta$-amyloid - $_{42}$
Pattern 3

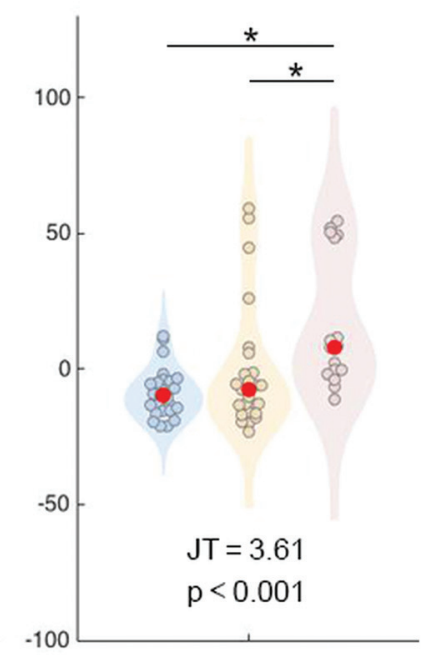

Nuclear DNA

$\square \mathrm{HC} \square \mathrm{PD}_{\mathrm{E}} \square \mathrm{PD}_{\mathrm{L}} \bigcirc$ median

FIG 2. Stage-specific patterns of plasma biomarkers. Violin plots demonstrate residual values of each biomarker adjusted for age and sex in healthy controls (blue), patients with $\mathrm{PD}_{\mathrm{E}}$ (yellow), and $\mathrm{PD}_{\mathrm{L}}$ (pink). Red circles of in the violin plots show the median value of each plasma biomarker of each study group. Asterisks indicate statistically significant differences among study groups using pair-wise Mann-Whitney $U$ tests (FDR-corrected, $P<.05$ ). JT indicates Jonckheere-Terpstra test.

\section{Stage-Specific Pattern of Gray Matter Volume}

Most regions in GMV analyses passed the Jonckheere-Terpstra test $\left(P_{\mathrm{FDR}}<.05\right)$; however, those eliminated included part of the frontal, parietal, and occipital lobes; the thalamus; and the insula. Post hoc tests further subdivided regional GMV patterns into 3 categories (Fig 3). Pattern 1 showed significantly lower GMVs in the $\mathrm{PD}_{\mathrm{E}}$ and $\mathrm{PD}_{\mathrm{L}}$ groups compared with controls, but no difference between the $\mathrm{PD}_{\mathrm{E}}$ and $\mathrm{PD}_{\mathrm{L}}$ groups in the right middle-frontal, left-lingual, and bilateral fusiform gyri; left hippocampus; and cerebellum. Pattern 2 featured progressive GMV atrophy with $\mathrm{PD}$ progression in the gyri including the bilateral precentral, postcentral, parahippocampal, left superior-temporal, right temporal, right superior-frontal, and left cingulate gyri in the occipital lobe and a small part of the bilateral cerebellum. In pattern 3, GMV was significantly lower in the $\mathrm{PD}_{\mathrm{L}}$ group compared with controls and the $\mathrm{PD}_{\mathrm{E}}$ group, but similar between controls and the $\mathrm{PD}_{\mathrm{E}}$ group, in the left middle-frontal, superiorfrontal, temporal, and posterior cingulate gyri and in the bilateral amygdala, caudate nucleus, and putamen.

\section{Associations of Stage-Specific Patterns between Plasma Biomarkers and Weighting Scores of GMVs}

Plasma T-tau exhibited a moderately negative correlation with GMV atrophy in all 3 patterns $\left(\rho=-0.331, P_{\mathrm{FDR}}=.006\right.$; $\left.\rho=-0.334, P_{\mathrm{FDR}}=.005 ; \rho=-0.410, P_{\mathrm{FDR}}=.001\right)($ Fig 4$)$, whereas plasma $\alpha$-synuclein showed a moderately negative correlation with only pattern 1 GMV atrophy $\left(\rho=-0.390, P_{\mathrm{FDR}}=\right.$ .001). Meanwhile, plasma nDNA showed a moderately negative correlation with only pattern 3 GMV atrophy $(\rho=-0.333$, $\left.P_{\mathrm{FDR}}=.005\right)$.

\section{Associations of Disease Severity, Cognitive Performance, Plasma Biomarkers, and Weighting Scores of GMVs}

UPDRS II and total score were positively correlated with plasma nDNA levels $\left(\rho=0.515, P_{\mathrm{FDR}}<.001 ; \rho=0.449, P_{F D R}=.003\right)$ (Fig 4 ). The MMSE performance was negatively correlated with T-tau, $\alpha$-synuclein, and nDNA levels $\left(\rho=-0.263, P_{F D R}=.029\right.$; $\left.\rho=-0.341, P_{F D R}=.004 ; \rho=-0.241, P_{F D R}=.046\right)$, while the CASI score was negatively correlated with $\alpha$-synuclein and nDNA levels $\left(\rho=-0.328, P_{F D R}=.006 ; \rho=-0.285, P_{F D R}=\right.$ .018). However, misfolding protein levels exhibited no correlations with disease severity. The GMV atrophy pattern 2 correlated with the UPDRS II, HY stage, and ADL $(\rho=-0.378$, $\left.P_{F D R}=.013 ; \rho=-0.379, P_{F D R}=.013 ; \rho=0.412, P_{F D R}=.007\right)$. Pattern 3 correlated with UPDRS II and III, and total score, HYstage, and ADL score $\left(\rho=-0.412, P_{F D R}=.007 ; \rho=-0.374\right.$, $P_{F D R}=.015 ; \rho=-0.409, P_{F D R}=.007 ; \rho=-0.451, P_{F D R}=.004$; $\rho=0.426, P_{F D R}=.005$ ) (Fig 4). Patterns 1,2 , and 3 also correlated with the MMSE $\left(\rho=0.396, P_{F D R}=.001 ; \rho=0.403\right.$, $\left.P_{F D R}=.001 ; \rho=0.396, P_{F D R}=.001\right)$. Patterns 1 and 3 correlated with the CASI $\left(\rho=0.274, P_{F D R}=.023 ; \rho=0.249, P_{F D R}=.039\right)$.

\section{DISCUSSION}

The results of this study reveal that changes in protienopathy and inflammation biomarkers are associated with GMV atrophy during PD progression. This finding indicates that elucidation of the associations among plasma biomarkers, GMV changes, and clinical status in PD may facilitate earlier diagnosis and improve prognostic accuracy.

Both the $\mathrm{PD}_{\mathrm{E}}$ and $\mathrm{PD}_{\mathrm{L}}$ groups had higher plasma levels of $\alpha$-synuclein, T-tau, and $\mathrm{A} \beta_{42}$ compared with the HCs, while 

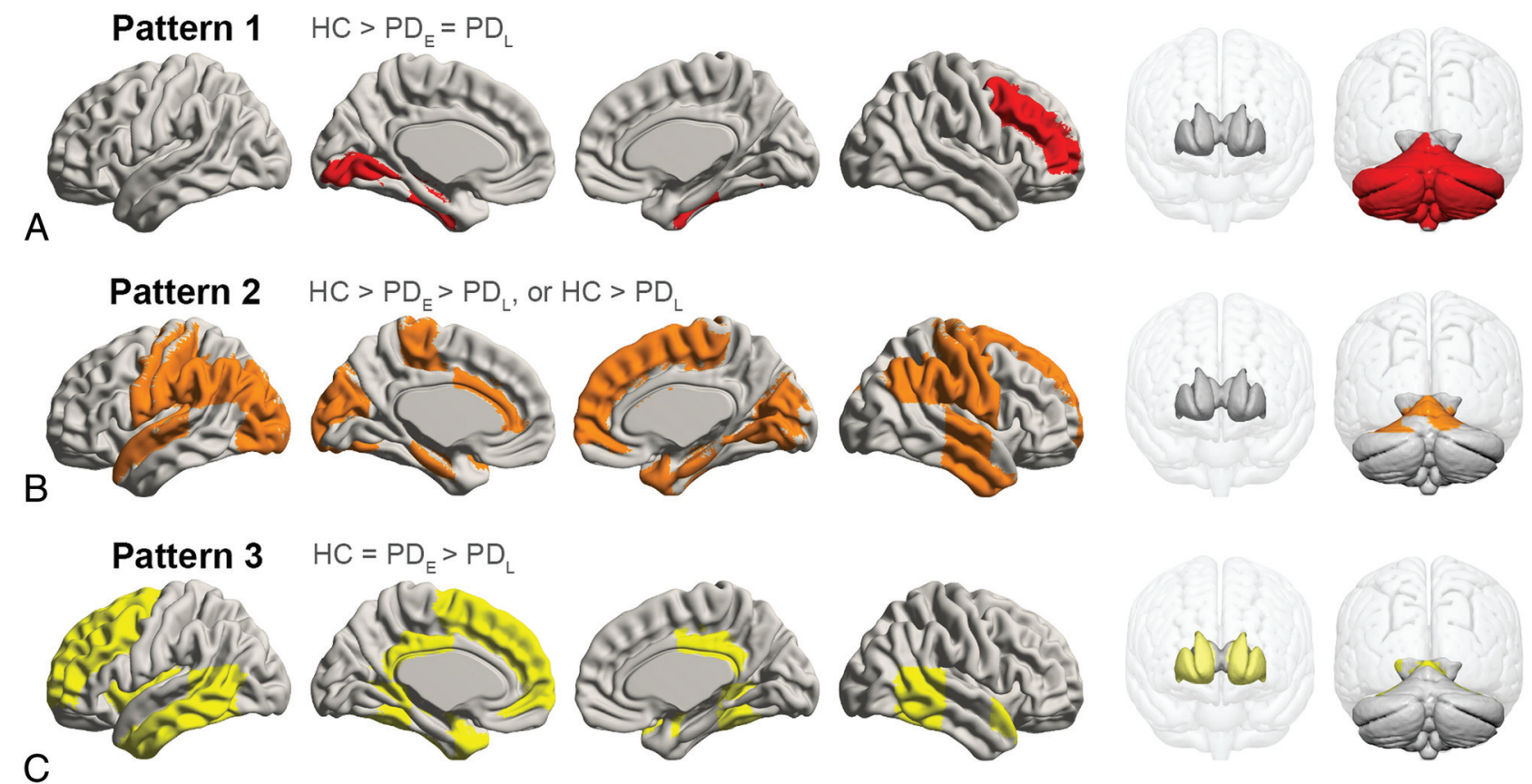

FIG 3. Spatial regions of stage-specific gray matter volume patterns. A, Pattern 1, red. B, Pattern 2, orange. C, Pattern 3, yellow.

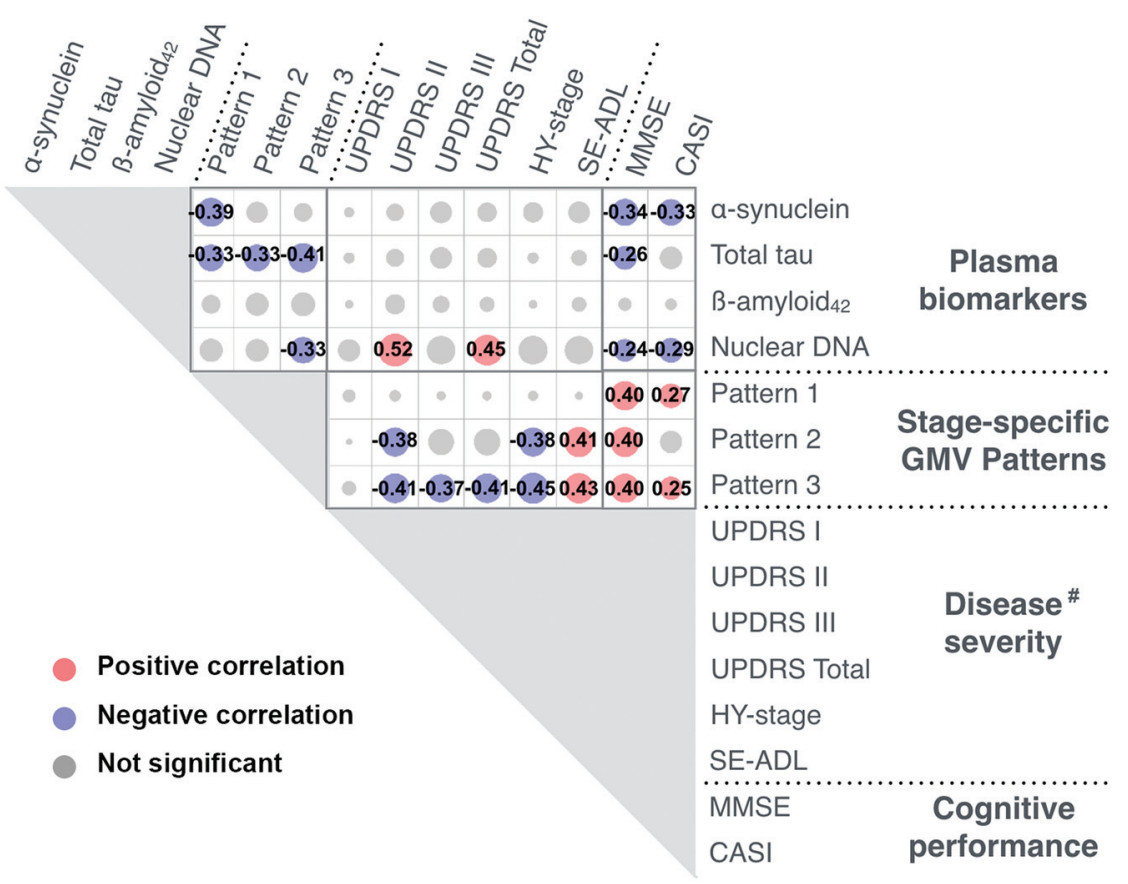

FIG 4. Associations among plasma biomarkers, weighting scores of stage-specific GMV patterns, disease severity, and cognitive performance. The hash tag indicates Spearman rank-order correlation analyses between disease severity and peripheral plasma biomarkers/weighting-score of stage-specific GMV patterns were conducted in patients with PD ( $P D_{E}$ and $\left.P D_{L}\right)$. SE-ADL indicates Schwab and England Activities of Daily Living scale.

nDNA levels were higher in the $\mathrm{PD}_{\mathrm{L}}$ than in $\mathrm{PD}_{\mathrm{E}}$ or HCs. Of note, levels of $\alpha$-synuclein, T-tau, and A $\beta_{42}$ were not associated with disease severity, and only UPDRS II and total score correlated with nDNA levels. GMV atrophy is pervasive as PD severity increases and can be temporally classified into 3 distinct patterns.
These results are consistent with the hypothesis that $\alpha$-synuclein, which is associated with GMV atrophy in the frontal lobe, hippocampus, and cerebellum, plays a potential pathogenic role in early $\mathrm{PD}$, while $\mathrm{nDNA}$ may signify GMV atrophy in the late stage of PD. Most important, the plasma T-tau level is a cardinal marker of characteristic brain degeneration patterns in different stages of PD.

The elevated $\alpha$-synuclein and T-tau levels noted in patients with PD herein are consistent with those in previous reports. $^{2-5} \alpha$-synuclein is pathognomonic in the etiology of PD and is found in both CSF and plasma after exocytosis from neurons. Our findings support the growing evidence that elevated plasma $\alpha$-synuclein offers potential as a biomarker to identify patients with PD. ${ }^{18}$ In a mouse model study, radiolabeled $\alpha$-synuclein from an intracerebroventricular injection was diverted to the blood via exosome efflux. ${ }^{10}$ Higher plasma levels of $\alpha$-synuclein in PD may be partially attributable to increased exosomal elimination of toxic $\alpha$-synuclein buildup from the brain to peripheral blood. Previous studies have demonstrated an inverse relationship between $\alpha$-synuclein levels in CSF versus plasma. ${ }^{32}$ This observation may reflect the deteriorating ability of the central nervous system to clear $\alpha$-synuclein and increased sequestration of pathologic 
soluble $\alpha$-synuclein in the brain, with consequently lower levels in CSF. However, further research is needed to clarify precisely how $\alpha$-synuclein is transported from the central nervous system to peripheral blood.

Numerous $\mathrm{A} \beta$ plaques and tau-containing neurofibrillary tangles develop in up to $50 \%$ of patients with $\mathrm{PD}_{\mathrm{L}}$, which may act synergistically with $\alpha$-synuclein pathology to worsen prognosis. However, current data on CSF tau levels in PD are inconclusive. ${ }^{32}$ Our study results are consistent with a recent report of a high plasma T-tau level in parkinsonism, indicating a potential role for plasma tau IMR in screening for neurodegenerative diseases. ${ }^{27}$ Plasma $\mathrm{A} \beta_{42}$, which was also elevated in our PD cohorts, has been extensively studied in CSF. ${ }^{32}$ There is evidence that lower baseline CSF $\mathrm{A} \beta_{42}$ may predict subsequent cognitive decline and dementia in patients with PD. ${ }^{12,33}$ Although misfolded $\alpha$-synuclein aggregation is considered a critical mechanism, the pathophysiology of PD is multifactorial. The comorbid associations of $\mathrm{A} \beta_{42}$ and tau deposition may be associated with cognitive deficits in PD. ${ }^{34}$ In terms of pathogenesis, amyloid- $\beta$ ( $\mathrm{A} \beta_{40}$ and $\mathrm{A} \beta_{42}$ ) is secreted by many types of cells and is normally found in the CSF; notably, studies have shown that $\mathrm{A} \beta_{42}$ can induce neuronal death. ${ }^{35,36}$ Thus, the elevated $\mathrm{A} \beta_{42}$ in this study indicates that potentially neurotoxic $\mathrm{A} \beta_{42}$ indeed increased in patients with $\mathrm{PD} .^{34-36}$

The $\alpha$-synuclein, T-tau, and $\mathrm{A} \beta_{42}$ levels in our study neither differentiated between $\mathrm{PD}_{\mathrm{E}}$ and $\mathrm{PD}_{\mathrm{L}}$ nor correlated with disease severity; however, $\alpha$-synuclein and T-tau have been correlated with cognitive function, possibly implicating AD neuropathology in the cognitive deterioration of patients with PD. Thus, further IMR assay investigation of plasma misfolding-associated proteins may identify potential biomarkers for diagnosing PD and evaluating related cognitive deficits. ${ }^{11}$

Although progressive GMV atrophy has been well-documented in patients with PD, the topographic disposition during the disease course was hitherto unclear. Our systematic investigation revealed alterations in the right frontal lateral lobe, hippocampus, and bilateral cerebellum, in both $\mathrm{PD}_{\mathrm{E}}$ and $\mathrm{PD}_{\mathrm{L}}$ in pattern 1, with no significant differences between groups. The frontostriatal and limbic circuits, which are involved in dopaminergic activity, are commonly affected in early-stage PD but are not associated with a higher risk of subsequent dementia. ${ }^{37} \mathrm{~A}$ recent study also demonstrated widespread early changes in the structure and function of motor and cognitive cerebellar regions in patients with $\mathrm{PD},{ }^{38}$ though Braak staging does not specifically address such cerebellar features. These neuroanatomic changes are also correlated with plasma $\alpha$-synuclein and T-tau levels, corroborating the role of synucleinopathy in PD and further indicating the possibility of tau co-pathology. Regardless of the cause of neuronal death, the areas in pattern 1 affected from $\mathrm{PD}_{\mathrm{E}}$ to $\mathrm{PD}_{\mathrm{L}}$ implicate the involvement of dopamine neuron substrates during the development of parkinsonian symptoms. Our results indicate that plasma $\alpha$-synuclein may play a major pathophysiologic role in early GMV atrophy, particularly in the frontal lobe, hippocampus, and cerebellum. Therefore, $\alpha$-synuclein-associated anatomies could act as markers of PD status.

The spatiotemporal progressive atrophic pattern 2 largely involves the posterior neocortices. Different neurotransmitters could also malfunction secondary to dopaminergic neuron loss or dopaminergic treatment, which may account for subsequent nonmotor impairment. ${ }^{39}$ Of note, this atrophy pattern was associated with plasma $\mathrm{T}$-tau but not $\mathrm{A} \beta_{42}$. A study of $\mathrm{AD}$ has reported a weak association of $\mathrm{A} \beta$ with neurodegeneration, despite a strong link between $\mathrm{A} \beta$ and tau; however, tau was associated with brain atrophy. ${ }^{40}$ Correlations with higher plasma tau in all 3 patterns that we identified suggest that this biomarker is associated with characteristic topographic degeneration throughout the course of PD. Furthermore, PET studies in patients with dementia with Lewy bodies and $\mathrm{PD}^{41}$ have revealed abnormal tau binding distributions similar to those noted in our pattern 2 GMV atrophy. This finding suggests that the neuropathologic processes that drive tau deposition in PD reflect the same regional vulnerabilities as those driving tau deposition in $\mathrm{AD}$. Thus, pattern 2 atrophy and the associated cognitive symptoms may be effective PD staging biomarkers.

In this study, the pattern 3 areas are mainly located in areas least involved in PD progression until the late stage. Their topographic distribution was consistent with those neocortices less affected in Braak stages V-VI, for both $\alpha$-synuclein and tau pathology. ${ }^{1}$ We further demonstrated associations between plasma tau and nDNA levels. Oxidative stress and elevated neuroinflammation are considered important etiologies in $\mathrm{PD}^{19}$ and can be revealed by a marker of cell death, such as plasma DNA levels. The breakdown of the blood-brain barrier may release DNA into the plasma, which has been shown to correlate with structural changes in early-onset PD. ${ }^{42}$ Although prion transfection is the prevailing hypothesis in PD pathogenesis, there is growing evidence suggesting that misfolded protein accumulation is necessary but not sufficient to cause neurodegeneration, because the neurodegeneration occurs only in the environment of a specific innate immune response. ${ }^{43}$ Our plasma nDNA and tau findings are indicative of neurodegeneration in $\mathrm{PD}_{\mathrm{L}}$.

Notwithstanding the strengths of this study, our findings should be interpreted in light of some limitations. Our study groups were selected to be as homogeneous as possible, and the relatively small age- and sex-matched PD cohorts may not fully represent the disease spectrum of $\mathrm{PD}$. Given the exploratory design of this study, analyses were based on a simple grouping scheme according to the HY stage, which, though acceptable for general evaluation of disease status, may preclude investigation of subgroup patterns corresponding to different clinical phenotypes with similar disease severity. Elucidating potential relationships between stage-specific atrophic patterns and plasma biomarkers was also hindered by the cross-sectional design. Longitudinal studies may provide further insight into the vulnerability of distinct brain regions as well as their atrophy trajectories during the course of PD.

\section{CONCLUSIONS}

As with most neurodegenerative diseases, the clinicopathology of PD involves complex and dynamic interactions between several underlying etiologic mechanisms. This study revealed that multiple markers of proteinopathy and inflammation are associated with regional GMV atrophy. We herein identified plasma biomarkers that characterize stage-specific GMV atrophy patterns 
and clinical features in PD. Such biomarkers, possibly identified from different modalities and following validation in large, longitudinal cohorts may facilitate early diagnosis and improved accuracy of prognostic evaluation.

\section{ACKNOWLEDGMENTS}

This research was financially supported by the Brain Research Center, National Yang Ming Chiao Tung University from The Featured Areas Research Center Program within the framework of the Higher Education Sprout Project by the Ministry of Education (MOE) in Taiwan.

Dr David Neil, PhD, of Full Universe Integrated Marketing provided professional editorial services, and his colleague Pei Chi Kuo assisted with project management for manuscript preparation.

\section{REFERENCES}

1. Braak H, Del Tredici K, Rüb U, et al. Staging of brain pathology related to sporadic Parkinson's disease. Neurobiol Aging 2003;24:197211 CrossRef Medline

2. Burke RE, Dauer WT, Vonsattel JP. A critical evaluation of the Braak staging scheme for Parkinson's disease. Ann Neurol 2008;64:485-91 CrossRef Medline

3. Braak H, Rub U, Jansen SE, et al. Cognitive status correlates with neuropathologic stage in Parkinson disease. Neurology 2005;64:1404-10 CrossRef Medline

4. Irwin DJ, White MT, Toledo JB, et al. Neuropathologic substrates of Parkinson disease dementia. Ann Neurol 2012;72:587-98 CrossRef Medline

5. Gerhard A, Pavese $\mathrm{N}$, Hotton G, et al. In vivo imaging of microglial activation with [11C](R)-PK11195 PET in idiopathic Parkinson's disease. Neurobiol Dis 2006;21:404-12 CrossRef Medline

6. Paola D, Domenicotti C, Nitti M, et al. Oxidative stress induces increase in intracellular amyloid beta-protein production and selective activation of betaI and betaII PKCs in NT2 cells. Biochem Biophys Res Commun 2000;268:642-46 CrossRef Medline

7. Petrou M, Dwamena BA, Foerster BR, et al. Amyloid deposition in Parkinson's disease and cognitive impairment: a systematic review. Mov Disord 2015;30:928-35 CrossRef Medline

8. Kotzbauer PT, Cairns NJ, Campbell MC, et al. Pathologic accumulation of alpha-synuclein and Abeta in Parkinson disease patients with dementia. Arch Neurol 2012;69:1326-31 CrossRef Medline

9. Mollenhauer B, Locascio JJ, Schulz-Schaeffer W, et al. $\alpha$-Synuclein and tau concentrations in cerebrospinal fluid of patients presenting with parkinsonism: a cohort study. Lancet Neurol 2011;10:23040 CrossRef Medline

10. Shi M, Liu C, Cook TJ, et al. Plasma exosomal alpha-synuclein is likely CNS-derived and increased in Parkinson's disease. Acta Neuropathol 2014;128:639-50 CrossRef Medline

11. Yang SY, Chiu MJ, Lin CH, et al. Development of an ultra-high sensitive immunoassay with plasma biomarker for differentiating Parkinson disease dementia from Parkinson disease using antibody functionalized magnetic nanoparticles. J Nanobiotechnology 2016;14:41 CrossRef Medline

12. Siderowf A, Xie SX, Hurtig H, et al. CSF amyloid \{beta\} 1-42 predicts cognitive decline in Parkinson disease. Neurology 2010;75:1055-61 CrossRef Medline

13. Kang JH, Irwin DJ, Chen-Plotkin AS, et al. Parkinson's Progression Markers Initiative. Association of cerebrospinal fluid beta-amyloid 1-42, T-tau, P-tau181, and alpha-synuclein levels with clinical features of drug-naive patients with early Parkinson disease. JAMA Neurol 2013;70:1277-87 CrossRef Medline
14. Atik A, Stewart T, Zhang J. $\alpha$-synuclein as a biomarker for Parkinson's disease. Brain Pathol 2016;26:410-18 CrossRef Medline

15. Yang CC, Yang SY, Chieh JJ, et al. Biofunctionalized magnetic nanoparticles for specifically detecting biomarkers of Alzheimer's disease in vitro. ACS Chem Neurosci 2011;2:500-505 CrossRef Medline

16. Chiu MJ, Chen YF, Chen TF, et al. Plasma tau as a window to the brain-negative associations with brain volume and memory function in mild cognitive impairment and early Alzheimer's disease. Hum Brain Mapp 2014;35:3132-42 CrossRef Medline

17. Chiu MJ, Yang SY, Horng HE, et al. Combined plasma biomarkers for diagnosing mild cognition impairment and Alzheimer's disease. ACS Chem Neurosci 2013;4:1530-36 CrossRef Medline

18. Lin CH, Yang SY, Horng HE, et al. Plasma $\alpha$-synuclein predicts cognitive decline in Parkinson's disease. J Neurol Neurosurg Psychiatry 2017;88:818-24 CrossRef Medline

19. Makin S. Pathology: the prion principle. Nature 2016;538:S13-16 CrossRef Medline

20. Pavlov VA, Tracey KJ. Neural circuitry and immunity. Immunol Res 2015;63:38-57 CrossRef Medline

21. Hughes AJ, Daniel SE, Kilford L, et al. Accuracy of clinical diagnosis of idiopathic Parkinson's disease: a clinico-pathological study of 100 cases. J Neurol Neurosurg Psychiatry 1992;55:181-84 CrossRef Medline

22. Gasser T, Bressman S, Durr A, et al. State of the art review: molecular diagnosis of inherited movement disorders: Movement Disorders Society Task Force on molecular diagnosis. Mov Disord 2003;18:3-18 CrossRef Medline

23. Goetz CG, Poewe W, Rascol O, et al. Movement Disorder Society Task Force report on the Hoehn and Yahr staging scale: status and recommendations of the Movement Disorder Society Task Force on rating scales for Parkinson's disease. Mov Disord 2004;19:102028 CrossRef Medline

24. Bjornestad A, Tysnes OB, Larsen JP, et al. Reliability of three disability scales for detection of independence loss in Parkinson's disease. Parkinsons Dis 2016;2016:1941034 CrossRef Medline

25. Folstein MF, Folstein SE, McHugh PR. "Mini-mental state". A practical method for grading the cognitive state of patients for the clinician. J Psychiatr Res 1975;12:189-98 CrossRef Medline

26. Chang CC, Liu JS, Chang YY, et al. (99m)Tc-ethyl cysteinate dimer brain SPECT findings in early stage of dementia with Lewy bodies and Parkinson's disease patients: a correlation with neuropsychological tests. Eur J Neurol 2008;15:61-66 CrossRef Medline

27. Yang SY, Chiu MJ, Chen TF, et al. Analytical performance of reagent for assaying tau protein in human plasma and feasibility study screening neurodegenerative diseases. Sci Rep 2017;7:9304 CrossRef Medline

28. Lin CH, Yang SY, Horng HE, et al. Plasma biomarkers differentiate parkinson's disease from atypical parkinsonism syndromes. Front Aging Neurosci 2018;10:123 CrossRef Medline

29. Wang HC, Yang TM, Lin WC, et al. The value of serial plasma and cerebrospinal fluid nuclear and mitochondrial deoxyribonucleic acid levels in aneurysmal subarachnoid hemorrhage. J Neurosurg 2013;118:13-19 CrossRef Medline

30. Zhang Y, Cabilio P. A generalized Jonckheere test against ordered alternatives for repeated measures in randomized blocks. Stat Med 2013;32:1635-45 CrossRef Medline

31. Pernet CR, Wilcox R, Rousselet GA. Robust correlation analyses: false positive and power validation using a new open source Matlab toolbox. Front Psychol 2012;3:606 CrossRef Medline

32. Magdalinou N, Lees AJ, Zetterberg H. Cerebrospinal fluid biomarkers in parkinsonian conditions: an update and future directions. $J$ Neurol Neurosurg Psychiatry 2014;85:1065-75 CrossRef Medline

33. Compta Y, Pereira JB, Rios J, et al. Combined dementia-risk biomarkers in Parkinson's disease: a prospective longitudinal study. Parkinsonism Rel Disord 2013;19:717-24 CrossRef Medline

34. Jellinger KA, Seppi K, Wenning GK, et al. Impact of coexistent Alzheimer pathology on the natural history of Parkinson's disease. J Neural Transm (Vienna) 2002;109:329-39 CrossRef Medline 
35. Zou K, Gong JS, Yanagisawa K, et al. A novel function of monomeric amyloid beta-protein serving as an antioxidant molecule against metal-induced oxidative damage. J Neurosci 2002;22:483341 Medline

36. Zou K, Kim D, Kakio A, et al. Amyloid beta-protein (Abeta)140 protects neurons from damage induced by Abeta1-42 in culture and in rat brain. J Neurochem 2003;87:609-19 CrossRef Medline

37. Uribe C, Segura B, Baggio HC, et al. Cortical atrophy patterns in early Parkinson's disease patients using hierarchical cluster analysis. Parkinsonism Relat Disord 2018;50:3-9 CrossRef Medline

38. O'Callaghan C, Hornberger M, Balsters JH, et al. Cerebellar atrophy in Parkinson's disease and its implication for network connectivity. Brain 2016;139:845-55 CrossRef Medline
39. Roy R, Niccolini F, Pagano G, et al. Cholinergic imaging in dementia spectrum disorders. Eur J Nucl Med Mol Imaging 2016;43:137686 CrossRef Medline

40. Jagust W. Imaging the evolution and pathophysiology of Alzheimer disease. Nat Rev Neurosci 2018;19:687-700 CrossRef Medline

41. Gomperts SN, Locascio JJ, Makaretz SJ, et al. Tau positron emission tomographic imaging in the Lewy body diseases. JAMA Neurol 2016;73:1334-41 CrossRef Medline

42. Chen YS, Chen $\mathrm{MH}, \mathrm{Lu} \mathrm{CH}$, et al. Associations among cognitive functions, plasma DNA, and white matter integrity in patients with earlyonset Parkinson's Disease. Front Neurosci 2017;11:9 CrossRef Medline

43. Alibhai J, Blanco RA, Barria MA, et al. Distribution of misfolded prion protein seeding activity alone does not predict regions of neurodegeneration. PLoS Biol 2016;14:e1002579 CrossRef Medline 